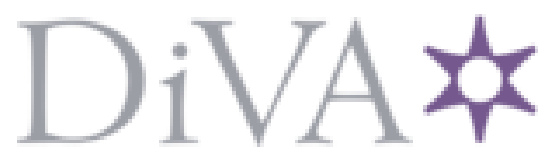

http://www.diva-portal.org

\title{
Postprint
}

This is the accepted version of a paper published in European Journal of Heart Failure. This paper has been peer-reviewed but does not include the final publisher proof-corrections or journal pagination.

Citation for the original published paper (version of record):

Carlsson, A., Larsson, A., Helmersson-Karlqvist, J., Lind, L., Ingelsson, E. et al. (2013)

Urinary kidney injury molecule 1 and incidence of heart failure in elderly men.

European Journal of Heart Failure, 15(4): 441-446

http://dx.doi.org/10.1093/eurjhf/hfs187

Access to the published version may require subscription.

N.B. When citing this work, cite the original published paper.

Permanent link to this version:

http://urn.kb.se/resolve?urn=urn:nbn:se:uu:diva- 188160 


\section{Urinary kidney injury molecule-1 and incidence of heart failure in elderly}

\section{men}

Axel C Carlsson ${ }^{1,2}$, Anders Larsson 3 , Johanna Helmersson-Karlqvist ${ }^{3}$, Lars

Lind $^{3}$, Erik Ingelsson ${ }^{4}$, Tobias E Larsson ${ }^{5}$, Johan Sundström³ ${ }^{3}$, and Johan

\section{Ärnlöv $2,6^{*}$}

${ }^{1}$ Centre for Family Medicine, Department of Neurobiology, Care Sciences and Society, Karolinska Institutet, Huddinge, Sweden; ${ }^{2}$ Department of Public Health and Caring Sciences/ Section of Geriatrics Uppsala University, Uppsala Sweden; ${ }^{3}$ Department of Medical Sciences, Uppsala University Hospital, Uppsala, Sweden; ${ }^{4}$ Department of Medical Epidemiology and Biostatistics, Karolinska Institutet, Stockholm, Sweden; ${ }^{5}$ Department of Clinical Science, Intervention and Technology, Karolinska Institutet, Stockholm, Sweden; ${ }^{6}$ School of Health and Social Studies, Dalarna University, Falun, Sweden

*Corresponding author: Johan Ärnlöv, Department of Public Health and Caring Sciences/Geriatrics, Uppsala Science Park, SE-75185 Uppsala, Sweden

Fax: +46-18-6117976; phone: +46-70-6077703

E-mail: johan.arnlov@ pubcare.uu.se

Words in abstract: 250

Number of words including tables, figures and references: 4590

Tables: 3 Figures: 2 References: 30 


\begin{abstract}
Objective There is growing recognition of the clinical importance of the cardiorenal syndrome - the bidirectional interplay between kidney and cardiac dysfunction. Yet, the role of kidney tubular damage in the development of heart failure is less studied. The objective was to investigate whether urinary kidney injury molecule (KIM)-1, a specific marker of tubular damage, predisposes to an increased heart failure risk.
\end{abstract}

Methods A community-based cohort study (Uppsala Longitudinal study of Adult Men [ULSAM]) of 565, 77-year-old men free from heart failure at baseline. Heart failure hospitalizations were used as outcome.

Results During follow-up (median 8.0 years) 73 participants were hospitalized for heart failure. In models adjusted for cardiovascular risk factors (age, systolic blood pressure, diabetes, smoking, body mass index, LDL/HDL-ratio, antihypertensive treatment, lipidlowering treatment, aspirin treatment, left ventricular hypertrophy and prevalent cardiovascular disease) and markers of kidney dysfunction and damage (cystatin C-based glomerular filtration rate (GFR) and urinary albumin/creatinine-ratio), higher urinary KIM1/creatinine ratio was associated with higher risk for heart failure (HR upper vs. lower tertile, $1.81 ; 95 \%$ CI 1.01 to $3.29 ; \mathrm{P}<0.05)$. Participants with a combination of low GFR $(<60$ $\mathrm{mL} / \mathrm{min} / 1.72 \mathrm{~m}^{2}$ ) and high KIM-1/creatinine (>128 ng/mmol) had a 3-fold increase in heart failure risk compared to participants with normal GFR and KIM-1 ( $<<0.001)$.

Conclusion Our findings suggest that kidney tubular damage predisposes to an increased risk for heart failure in the community. Further studies are needed to clarify the causal role of KIM-1 in the development of heart failure, and to evaluate the clinical utility of urinary KIM1 measurements.

Key Words: Heart failure, tubular damage, cohort study, kidney function, glomerular filtration rate 


\section{Introduction}

There is growing recognition of the clinical importance of the cardiorenal syndrome - the bidirectional interplay between diminished kidney function and cardiac dysfunction. ${ }^{1}$ It is well known that patients with heart failure are at a substantially increased risk to develop kidney failure. ${ }^{2}$ The opposite chain of events is also common; patients with overt kidney disease are at an increased risk for incident heart failure. ${ }^{3}$ In fact, recent studies show that even mild derangements of glomerular filtration rate (GFR) or albuminuria are associated with an increased risk for heart failure, ${ }^{4}$ suggesting that the cardiorenal-syndrome begin even prior to the development of clinically overt kidney- and cardiac dysfunction.

The specific role of kidney tubular damage in the interplay between the kidney and the heart is, however, less investigated. A few previous studies have shown that heart failure patients with signs of kidney tubular damage are at an increased risk of morbidity and mortality. 5 Yet, whether tubular damage is linked to an increased risk for heart failure in the community is unknown.

Kidney injury molecule 1 (KIM-1) is a trans-membrane protein that is highly expressed in epithelial cells in damaged regions of the proximal tubuli, ${ }^{7}$ and possibly also in the glomeruli. ${ }^{8}$ KIM-1 has been suggested to be involved in the modulation of tubular damage and repair in response to acute and chronic kidney injury. ${ }^{9,}$ Urinary levels of KIM-1 has been suggested as a clinically relevant biomarker of acute tubular injury, ${ }^{7}$ as it rises more rapidly and is more specific to tubular damage than albuminuria or low GFR. Yet, the utility of urinary KIM-1 as a prognostic marker of incident heart failure remains to be established.

In the present study, we hypothesized that individuals with early signs of renal tubular damage are at an increased risk of heart failure. Accordingly, the aim of this study was to 
investigate the association between urinary levels of KIM-1(KIM-1/creatinine ratio) and the incidence of heart failure hospitalizations in a community-based sample of elderly men. We also wanted to explore if this association was independent of cardiovascular risk factors, and other markers of kidney damage and dysfunction. 


\section{Methods}

\section{Study sample}

The design and selection criteria of the Uppsala Longitudinal study of Adult Men (ULSAM) have been described previously, ${ }^{4}$ and further details can be found on the internet at http://www.pubcare.uu.se/ULSAM. At the fourth examination cycle (1998-2002), 838 men (mean age 77.5 years) were investigated. Of these, urine samples were available in 627 individuals. Twenty-five participants were excluded due to a previous diagnosis of heart failure, 37 participants were excluded due to missing data on covariates leaving 565 individuals as the present study sample. All participants gave written informed consent, and the ethics committee of Uppsala University approved the study protocol.

\section{Clinical and biochemical evaluation at baseline}

Blood pressure was measured in the right arm with the subject in the supine position after resting for 10 minutes. The body mass index (BMI) was calculated as the individual's body weight divided by height squared $\left(\mathrm{kg} / \mathrm{m}^{2}\right)$. Venous blood samples were drawn in the morning after an overnight fast and stored at $-70^{\circ} \mathrm{C}$ until analysis. Total and HDL-cholesterol were measured by routine laboratory analysis. Plasma glucose concentrations were measured by the glucose dehydrogenase method (Gluc-DH, Merck, Darmstadt, Germany). Cystatin C was measured was measured by latex enhanced reagent (NLatex Cystatin C; Siemens, Deerfield, IL, USA) using a BN ProSpec ${ }^{\circledR}$ analyzer (Siemens) and used to estimate GFR. ${ }^{11}$ 24-Hour urine were collected, aliquoted and stored at $-70^{\circ}$ until analysis. Urinary KIM-1 levels were analyzed with the commercial sandwich ELISA kit in 100ul urine samples (DY1750 R\&D Systems, Minneapolis, MN, USA) using a validated assay. ${ }^{12}$ The inter-assay CV was $5 \%$ and the intra-assey CV was 4\%. The lowest level of detection (lowest standard point) was 31 $\mathrm{pg} / \mathrm{mL}$. Urine albumin was measured by nephelometry (Urine albumin, Siemens) using a BN 
ProSpec ${ }^{\circledR}$ analyzer (Siemens). Urine creatinine was analyzed with a modified kinetic Jaffe reaction on an Architect Ci8200® analyzer (Abbott, Abbot Park, IL, USA) and creatinine related urine albumin was calculated from the BN ProSpec ${ }^{\circledR}$ results. High-sensitive CRP measurements were performed by latex-enhanced reagent (Siemens) with the use of a BN ProSpec $^{\circledR}$ analyzer (Siemens). Electrographic left ventricular hypertrophy (ECG-LVH) was defined as high amplitude R-waves according to the revised Minnesota code, together with a left ventricular strain pattern. ${ }^{13}$ Prevalent cardiovascular disease at baseline was defined as a history of ischemic heart disease or cerebrovascular disease, or Q-, QS-complexes or left bundle-branch block in baseline ECG. Diabetes was defined as self-reported use of insulin or oral anti diabetic drugs in the questionnaire, or as having fasting plasma glucose above 7 $\mathrm{mmol} / \mathrm{L}$. Use of low-dose aspirin, treatment for blood lipids (mostly statins), and treatment for hypertension was established using a questionnaire. Markers of inflammation and oxidative stress were analyzed as previously described. ${ }^{14}$

\section{Outcome}

The classification of heart failure events during follow-up have been described in detail previously. ${ }^{15}$ In short, as a possible diagnosis of heart failure, we considered hospitalizations for ICD10 heart failure codes I50 and hypertensive heart disease with heart failure, I11.0 from the Swedish Hospital Discharge Register. The medical records from the relevant hospitalizations until December 31, 2008, were reviewed by three physicians (E.I., L.L. and J.S.), who, blinded to the baseline data, classified the cases as definite, questionable, or miscoded. Only the definite heart failure cases, defined as a combination of symptoms of heart failure and objective signs of cardiac dysfunction at rest, were used as outcome in the present study. The classification relied on the definition proposed by the European Society of Cardiology. ${ }^{16}$ 


\section{Statistical analysis}

Mean values and standard deviations were calculated for all continuous variables at baseline. The association between tertiles of urinary KIM-1/creatinine and the incidence of heart failure hospitalizations was investigated using Cox proportional hazard regression. The lower tertile was used as referent. Adjustments were made using the following multivariable models: Model A: age-adjusted (age was adjusted for in all models by using age as the timeline).

Model B: adjusted for age and established cardiovascular risk factors (CVD at baseline, antihypertensive treatment, blood lipid lowering treatment, low-dose aspirin, current smoking and diabetes, left ventricular hypertrophy [as binary variables], systolic blood pressure, BMI, total cholesterol and HDL cholesterol [as continuous variables]).

Model C: adjusted for age, established cardiovascular risk factors (as shown in model B), and markers of kidney damage and dysfunction (GFR and urinary albumin/creatinine ratio). In secondary analyses, we also added a marker of inflammation, c-reactive protein [CRP], to multivariable model C. Moreover, as different types of anti-hypertensive treatment such as diuretics or renin-angiotensin-aldoesterone inhibition have been shown to influence urinary KIM-1 levels, ${ }^{17,18}$ and as KIM-1 has been suggested to be involved in the development of hypertensive kidney disease, ${ }^{19}$ we performed secondary analyses where we replaced the antihypertensive treatment variable in the model B with use of ACE inhibitors, AII-blockers, alpha-blockers, beta-blockers, calcium antagonists or diuretics and where the office blood pressure variable was replaced by systolic and diastolic 24 -hour blood pressure.

Proportional hazards assumptions were confirmed by Schoenfeld's tests. To gain insights into potential nonlinearity of the associations, we examined Cox regression models by using penalized splines with three degrees of freedom. 
Additionally, we performed tests for effect modification by diabetes, hypertension, prevalent cardiovascular disease, microalbuminuria and GFR by including multiplicative interaction terms with these variables and urinary KIM-1.

To evaluate the interplay between KIM-1 and glomerular filtration rate in development of heart failure we divided the participants in the following 4 groups:

1. Participants with normal GFR ( $\geq 60 \mathrm{~mL} / \mathrm{min} / 1.72 \mathrm{~m}^{2}$ ) and normal KIM-1 (below upper tertile, $<128 \mathrm{ng} / \mathrm{mmol}$ [reference group])

2. Participants with normal GFR ( $\left.>60 \mathrm{~mL} / \mathrm{min} / 1.72 \mathrm{~m}^{2}\right)$ and high KIM-1 $(\geq 128 \mathrm{ng} / \mathrm{mmol})$

3. Participants with low GFR ( $\left.<60 \mathrm{~mL} / \mathrm{min} / 1.72 \mathrm{~m}^{2}\right)$ and normal KIM-1 $(<128 \mathrm{ng} / \mathrm{mmol})$

4. Participants with both low GFR $\left(<60 \mathrm{~mL} / \mathrm{min} / 1.72 \mathrm{~m}^{2}\right)$ and high $\mathrm{KIM}-1(\geq 128$ $\mathrm{ng} / \mathrm{mmol})$

To limit the possibility of reverse causation as an explanation of our findings we performed analyses after exclusion of participants that experienced a heart failure event during the first two years of follow-up $(n=10)$. For the same reason, we also added plasma N-terminal brain natriuretic peptide (Nt-proBNP) taken at a re-examination 6 years prior to baseline to multivariable model C.

The population-attributable risk proportion was calculated as follows: $\mathrm{p}^{*}\left[\left(\mathrm{HR}_{\text {exposed }^{-}}\right.\right.$ $\left.\left.\mathrm{HR}_{\text {unexposed }}\right) / \mathrm{HR}_{\text {exposed }}\right]$, where $\mathrm{p}$ is the proportion of cases that were exposed and $\mathrm{HR}$ is the hazard ratio adjusted for the covariates in multivariable model with three kidney function components, GFR<60, ACR $<3.0$ and the upper tertile of KIM-1/creatine ratio. ${ }^{20}$

A two-sided $p$-value $<0.05$ was regarded as significant in all analyses. The statistical software package STATA 11.2 (Stata corp, College Station, Texas, USA) was used for all analyses. 


\section{Results}

Baseline characteristics are shown in Table 1. The Spearman correlation coefficient between KIM-1/creatinine and GFR was $-0.14(\mathrm{p}<0.001)$, and between KIM-1/creatinine and albumin/creatinine was $0.40(\mathrm{p}<0.001)$ at baseline. During follow-up (median 8.0 years, range 0.2-10.8 years), 73 individuals were hospitalized for heart failure (incidence rate 1.81/100 person-years at risk). The incidence rates for heart failure in tertiles of KIM-1/creatinine are shown in Table 2.

\section{Cox regression models}

In Cox proportional hazard models, higher urinary levels of KIM-1/creatinine were significantly associated with an increased risk for heart failure. The associations were only mildly effected by adjustments for age, cardiovascular risk factors, GFR, and urinary albumin/creatinine ratio (Model A-C, Table 2). Participants with KIM-1/creatinine levels in the upper tertile were at almost twice the risk for heart failure in multivariable models A-C using the lower tertile as referent (Table 2). The spline curve suggests no major increase in risk above the upper tertile (Figure 1). The excess risk is also illustrated in Figure 2, where KIM-1/creatinine levels in the upper tertile were associated with a higher risk of incident heart failure.

In secondary analyses, the association between KIM-1/creatinine and heart failure incidence remained significant when replacing the hypertension treatment variable with specific antihypertensive treatments and the office systolic blood pressure variable with 24-hour systolic and diastolic blood pressure in Model B (HR for upper tertile of KIM-1/creatinine, $1.84,95 \%$ CI 1.02-3.32, $\mathrm{p}=0.04)$. Moreover, the association between urinary KIM1/creatinine and heart failure incidence were still significant after adding CRP to 
multivariable model C (HR for upper tertile of KIM-1/creatinine,1.82, 95 \% CI, 1.01-3.29, $\mathrm{p}=0.046)$.

The association between urinary KIM-1 and heart failure incidence was attenuated after excluding participants who experienced a heart failure event during the first two years of follow-up (Model C: HR for upper tertile of KIM-1/creatinine, 1.60, 95\% CI 0.83-3.08, $\mathrm{p}=0.16$ ). Also, the results were no longer significant when Nt-proBNP were added to multivariable model C (HR for upper tertile of KIM-1 1.55, 95\% CI 0.84-2.85, $\mathrm{p}=0.16$ ).

When three different markers of kidney pathology, KIM-1, GFR and urinary albumin/creatinine ratio, were added to model B, only KIM-1 and GFR predicted heart failure (HR for upper tertile of KIM-1/creatinine 1.82, 95\% CI 1.01-3.29. $\mathrm{P}=0.046$; GFR HR per SD increase $0.6295 \%$ CI $0.48-0.81, \mathrm{p}<0.001$; and albumin/creatinine ratio HR per SD increase $0.9895 \%$ CI 0.96-1.01, $\mathrm{p}=0.26)$.

As seen in Table 3, participants with low GFR $\left(<60 \mathrm{~mL} / \mathrm{min} / 1.72 \mathrm{~m}^{2}\right)$ and high KIM-1 ((upper tertile) $>128 \mathrm{ng} / \mathrm{mmol}$ ) had a more then 3 -fold increase in risk for heart failure using individuals with normal KIM-1 and normal GFR as referent.

The population attributable risk proportion for the development of heart failure was $19 \%$ (95 $\%$ CI 0 - $29 \%, \mathrm{p}<0.05)$ for KIM-1 levels above the upper tertile (>128 ng/mmol), and $15 \%$ $(95 \% \mathrm{CI} 4-22 \%)$ for $\mathrm{GFR}<60 \mathrm{~mL} / \mathrm{min} / 1.72 \mathrm{~m}^{2}$.

The multiplicative interaction terms for diabetes, hypertension, prevalent cardiovascular disease, microalbuminuria or GFR were not significant ( $p>0.44$ for all). 


\section{Discussion}

In the present community-based cohort study of elderly men, higher urinary levels of KIM-1 were associated with an increased risk of incident heart failure hospitalizations. The association between KIM-1 and heart failure incidence remained robust after adjustments for age, established risk factors, albuminuria and GFR. Participants who had a combination of high KIM-1 and low GFR appeared to have the highest heart failure risk, indicating that different aspects of kidney pathology may portray additive prognostic information.

\section{Comparison with the literature}

Although several previous studies have shown a close relationship between different aspects of kidney pathology, such as low glomerular filtration rate and albuminuria, and the development of heart failure ${ }^{1}$, data on the interplay between kidney tubular damage and heart failure is scarce. In a few recent longitudinal studies in heart failure patients, higher levels of KIM-1 and other markers of tubular damage have been associated with adverse outcomes. ${ }^{5,6}$ KIM-1 has also been shown to be associated with decreased left ventricular function. ${ }^{21}$ However, we are aware of no previous studies that have reported the association between urinary KIM-1 and the risk of incident heart failure in the community.

\section{Potential mechanisms}

Previous studies indicate protective functions for KIM-1 in acute kidney injury and damaging functions in chronic kidney disease. ${ }^{7,9,22}$ However, the various functions of KIM-1 are incompletely understood and are currently being unravelled.

Although this is an observational study, and in such we cannot deem any finding causal, there are several potential mechanisms that may explain the association between KIM-1 levels and incident heart failure. Several lines of evidence indicate that the higher urinary levels of KIM- 
1 may reflect increased fluid retention, perhaps caused by cardiac dysfunction: Urinary KIM1 levels have been shown to be closely associated with changes in volume status induced by diuretic withdrawal and reinstitution in heart failure patients ${ }^{17}$ and correlations between higher levels of KIM-1 and decreased left ventricular systolic function as well as severity of New York Heart Association (NYHA) class has been reported. ${ }^{21}$ Moreover, urinary KIM-1 has been suggested to be a biomarker for activation of the renin-angiotensin-aldosteron system, ${ }^{19}$ which is an important regulator of volume status and a key underlying factor in the pathophysiology of heart failure. This notion, that our results could be explained by reverse causation due to sub-clinical cardiac dysfunction or undetected heart failure at baseline, is supported by the finding that the association between urinary KIM-1 and heart failure incidence were attenuated after exclusion of the participants who developed heart failure during the first two years of follow-up. This is further supported by the fact that when NtproBNP, taken 6 years prior to baseline, were added as to multivariable model $\mathrm{C}$, the association between KIM-1 and heart failure was also attenuated. Unfortunately, we are not able to firmly resolve the issue of reverse causation in the present study as no echocardiographic examination or natriuretic peptide measurements were performed at baseline.

Kidney tubular damage have also been shown to be associated with several of the major conditions that predispose to an increased risk for heart failure such as hypertension, ${ }^{19}$ diabetes,${ }^{23}$ dyslipidemia,${ }^{24}$, low glomerular filtration rate,${ }^{25}$ microalbuminuria, ${ }^{26}$ and inflammation. ${ }^{27}$ We found KIM-1 levels to be correlated with glomerular filtration rate and microalbuminuria at baseline, yet, the association between KIM-1 and incident CHF was essentially unaltered after taking these factors into account in the multivariable models indicating that these are not major pathways that explain our findings. 
Higher urinary KIM-1 levels have been put forward as a biomarker for deterioration of kidney function, also in the community-based setting. ${ }^{28}$ Thus, another possible explanation of our findings is that the participants with higher KIM-1 levels at baseline had a steeper decline in renal function during follow-up which in turn led to a higher risk for heart failure.

Notably, KIM-1 is normally found at low or undetectable levels in the circulation and is therefore unlikely to directly influence cardiac remodeling and/or function. However, on a more speculative note, we cannot rule out that activation of KIM-1 could release other, yet unidentified, systemically available tubular proteins that directly promote cardiac dysfunction and subsequent development of heart failure.

\section{Clinical implications}

As KIM-1 expression is virtually absent in normal kidneys, but rise rapidly in response to tubular damage, ${ }^{29}$ KIM-1 has been put forward as a clinically useful marker for acute tubular kidney damage. ${ }^{9,30}$ Yet, the clinical utility of measuring urinary KIM-1 in heart failure patients or in the community remains to be thoroughly evaluated. The substantial population attributable risk proportion for heart failure associated with elevated KIM-1 levels indicates that kidney tubular damage may be an important component of the interplay between the kidney and the heart.

\section{Strengths and Limitations}

Strengths of the study include the community-based study population, the longitudinal study design with long follow-up and the detailed characterization of the study participants with regards to cardiovascular risk factors and markers of kidney damage and dysfunction measured in samples from 24-h urine collections. Furthermore, the heart failure diagnosis was 
validated which decreases the inclusion of false-positive cases. The main limitation of this study is that we only had access to data in elderly men, why extrapolations of these findings to women, other ethnicities, and other age-groups have to be done with caution. Moreover, the moderately sized study sample led to wider confidence intervals and consequently a greater uncertainty of the true level of the risk estimates. Another limitation is that no data on whether the heart failure events were of systolic or diastolic type were collected. Additional studies are needed to investigate this issue.

In conclusion, our community-based data indicate that a higher urinary level of KIM-1 is linked to an increased risk of incident heart failure. Further studies are needed to clarify the pathophysiological role of KIM-1 in the development of heart failure, and to evaluate the utility of measuring urinary KIM-1 in the clinical setting.

\section{Acknowledgements}

This study was supported by The Swedish Research Council (2006-6555), the Swedish Heart and Lung foundation, the Marianne and Marcus Wallenberg Foundation, Dalarna University and Uppsala University. The study was investigator initiated and driven. The authors report no conflicts of interests in connection with this study. 


\section{References}

1. Arnlov J. Diminished renal function and the incidence of heart failure. Curr Cardiol Rev 2009;5(3):223-7.

2. Damman K, Voors AA, Navis G, van Veldhuisen DJ, Hillege HL. The cardiorenal syndrome in heart failure. Prog Cardiovasc Dis 2011;54(2):144-53.

3. Hillege HL, Nitsch D, Pfeffer MA, Swedberg K, McMurray JJ, Yusuf S, Granger CB, Michelson EL, Ostergren J, Cornel JH, de Zeeuw D, Pocock S, van Veldhuisen DJ. Renal function as a predictor of outcome in a broad spectrum of patients with heart failure. Circulation 2006;113(5):671-8.

4. Ingelsson E, Sundstrom J, Lind L, Riserus U, Larsson A, Basu S, Arnlov J. Low-grade albuminuria and the incidence of heart failure in a community-based cohort of elderly men. Eur Heart J 2007;28(14):1739-45.

5. Damman K, Masson S, Hillege HL, Maggioni AP, Voors AA, Opasich C, van Veldhuisen DJ, Montagna L, Cosmi F, Tognoni G, Tavazzi L, Latini R. Clinical outcome of renal tubular damage in chronic heart failure. Eur Heart J 2011;32(21):2705-12.

6. Damman K, Voors AA, Hillege HL, Navis G, Lechat P, van Veldhuisen DJ, Dargie HJ. Congestion in chronic systolic heart failure is related to renal dysfunction and increased mortality. Eur J Heart Fail 2010;12(9):974-82.

$7 . \quad H u o$ W, Zhang K, Nie Z, Li Q, Jin F. Kidney injury molecule-1 (KIM-1): a novel kidney-specific injury molecule playing potential double-edged functions in kidney injury. Transplant Rev (Orlando) 2010;24(3):143-6.

8. Zhao X, Zhang Y, Li L, Mann D, Imig JD, Emmett N, Gibbons G, Jin LM. Glomerular expression of kidney injury molecule-1 and podocytopenia in diabetic glomerulopathy. American journal of nephrology 2011;34(3):268-80.

9. Waanders F, van Timmeren MM, Stegeman CA, Bakker SJ, van Goor H.

Kidney injury molecule-1 in renal disease. J Pathol 2010;220(1):7-16.

10. Vaidya VS, Ferguson MA, Bonventre JV. Biomarkers of acute kidney injury. Annu Rev Pharmacol Toxicol 2008;48:463-93.

11. Larsson A, Malm J, Grubb A, Hansson LO. Calculation of glomerular filtration rate expressed in $\mathrm{mL} / \mathrm{min}$ from plasma cystatin C values in $\mathrm{mg} / \mathrm{L}$. Scand J Clin Lab Invest 2004; 64(1):25-30.

12. Chaturvedi S, Farmer T, Kapke GF. Assay validation for KIM-1: human urinary renal dysfunction biomarker. International journal of biological sciences 2009;5(2):128-34. 13. Levy D, Larson MG, Vasan RS, Kannel WB, Ho KK. The progression from hypertension to congestive heart failure. JAMA 1996;275(20):1557-62.

14. Helmersson J, Arnlov J, Larsson A, Basu S. Low dietary intake of beta-carotene, alpha-tocopherol and ascorbic acid is associated with increased inflammatory and oxidative stress status in a Swedish cohort. Br J Nutr 2009;101(12):1775-82.

15. Ingelsson E, Arnlov J, Sundstrom J, Lind L. The validity of a diagnosis of heart failure in a hospital discharge register. Eur J Heart Fail 2005;7(5):787-91.

16. Guidelines for the diagnosis of heart failure. The Task Force on Heart Failure of the European Society of Cardiology. Eur Heart J 1995;16(6):741-51.

17. Damman K, Ng Kam Chuen MJ, MacFadyen RJ, Lip GY, Gaze D, Collinson PO, Hillege HL, van Oeveren W, Voors AA, van Veldhuisen DJ. Volume status and diuretic 
therapy in systolic heart failure and the detection of early abnormalities in renal and tubular function. J Am Coll Cardiol 2011;57(22):2233-41.

18. Waanders F, Vaidya VS, van Goor H, Leuvenink H, Damman K, Hamming I, Bonventre JV, Vogt L, Navis G. Effect of renin-angiotensin-aldosterone system inhibition, dietary sodium restriction, and/or diuretics on urinary kidney injury molecule 1 excretion in nondiabetic proteinuric kidney disease: a post hoc analysis of a randomized controlled trial. Am J Kidney Dis 2009;53(1):16-25.

19. Schupp N, Kolkhof P, Queisser N, Gartner S, Schmid U, Kretschmer A, Hartmann E, Oli RG, Schafer S, Stopper H. Mineralocorticoid receptor-mediated DNA damage in kidneys of DOCA-salt hypertensive rats. FASEB J 2011;25(3):968-78.

20. Rockhill B, Newman B, Weinberg C. Use and misuse of population attributable fractions. Am J Public Health 1998;88(1):15-9.

21. Jungbauer CG, Birner C, Jung B, Buchner S, Lubnow M, von Bary C, Endemann D, Banas B, Mack M, Boger CA, Riegger G, Luchner A. Kidney injury molecule1 and $\mathrm{N}$-acetyl-ss-D-glucosaminidase in chronic heart failure: possible biomarkers of cardiorenal syndrome. Eur J Heart Fail 2011.

22. Han WK, Bailly V, Abichandani R, Thadhani R, Bonventre JV. Kidney Injury Molecule-1 (KIM-1): a novel biomarker for human renal proximal tubule injury. Kidney Int 2002;62(1):237-44.

23. Nauta FL, Boertien WE, Bakker SJ, van Goor H, van Oeveren W, de Jong PE, Bilo H, Gansevoort RT. Glomerular and tubular damage markers are elevated in patients with diabetes. Diabetes Care 2011;34(4):975-81.

24. Ichimura T, Asseldonk EJ, Humphreys BD, Gunaratnam L, Duffield JS, Bonventre JV. Kidney injury molecule-1 is a phosphatidylserine receptor that confers a phagocytic phenotype on epithelial cells. J Clin Invest 2008;118(5):1657-68.

25. Damman K, Van Veldhuisen DJ, Navis G, Vaidya VS, Smilde TD, Westenbrink BD, Bonventre JV, Voors AA, Hillege HL. Tubular damage in chronic systolic heart failure is associated with reduced survival independent of glomerular filtration rate. Heart 2010;96(16):1297-302.

26. Nauta FL, Bakker SJ, van Oeveren W, Navis G, van der Heide JJ, van Goor H, de Jong PE, Gansevoort RT. Albuminuria, proteinuria, and novel urine biomarkers as predictors of long-term allograft outcomes in kidney transplant recipients. Am J Kidney Dis 2011;57(5):733-43.

$27 . \quad$ Ding H, He Y, Li K, Yang J, Li X, Lu R, Gao W. Urinary neutrophil gelatinaseassociated lipocalin (NGAL) is an early biomarker for renal tubulointerstitial injury in IgA nephropathy. Clin Immunol 2007;123(2):227-34.

28. Peralta CA, Katz R, Bonventre JV, Sabbisetti V, Siscovick D, Sarnak M, Shlipak MG. Associations of Urinary Levels of Kidney Injury Molecule 1 (KIM-1) and Neutrophil Gelatinase-Associated Lipocalin (NGAL) With Kidney Function Decline in the Multi-Ethnic Study of Atherosclerosis (MESA). American journal of kidney diseases : the official journal of the National Kidney Foundation 2012.

$29 . \quad$ Bonventre JV. Kidney injury molecule-1 (KIM-1): a urinary biomarker and much more. Nephrol Dial Transplant 2009;24(11):3265-8.

30. Vaidya VS, Niewczas MA, Ficociello LH, Johnson AC, Collings FB, Warram $\mathrm{JH}$, Krolewski AS, Bonventre JV. Regression of microalbuminuria in type 1 diabetes is associated with lower levels of urinary tubular injury biomarkers, kidney injury molecule-1, and N-acetyl-beta-D-glucosaminidase. Kidney international 2011;79(4):464-70. 
Table 1 Baseline characteristics of the 77-year old men

Variable

\begin{tabular}{ll}
\hline $\mathrm{N}$ & 565 \\
Age (years) & $77.5(0.76)$ \\
$\mathrm{BMI}\left(\mathrm{Kg} / \mathrm{m}^{2}\right)$ & $26.2(3.4)$ \\
Systolic blood pressure (mmHg) & $151(21)$ \\
Diastolic blood pressure & $81(9.4)$ \\
(mmHg) & \\
LDL/HDL ratio & $2.77(0.93)$ \\
GFR (mL/min/1.72m $\left.{ }^{2}\right)$ & $74.1(17)$ \\
Albumin/creatinine ratio & $0.7(0.3-2.0)$ \\
(mg/mmolcreatinine) & \\
KIM-1 (ng/mmol) & $824(492-$ \\
& $1262)$ \\
CVD at baseline & $130(23 \%)$ \\
Left ventricle hypertrophy & $164(29 \%)$ \\
Smoking & $40(7 \%)$ \\
Diabetes & $72(13 \%)$ \\
Antihypertensive medication & $258(46 \%)$ \\
Lipid lowering medication & $102(18 \%)$ \\
Low dose aspirin & $151(27 \%)$ \\
Alfa-blocker & $10(2 \%)$ \\
Diuretic & $80(14 \%)$ \\
Beta-blocker & $137(24 \%)$ \\
Calcium antagonist & $89(16 \%)$ \\
RAS blocker & $77(14 \%)$ \\
\hline Data
\end{tabular}

Data are mean \pm standard deviation for continuous variables (except for KIM-1 and Albumin/creatinine ratio where medians and inter quartile ranges are presented) and $\mathrm{n}(\%)$ for categorical variables.

BMI body mass index

LDL/HDL low density lipoprotein/high density lipoprotein

KIM-1 urinary levels of kidney injury molecule 1

GFR cyctatin $\mathrm{C}$ based estimation of glomerular filtration rate

CVD cardiovascular disease

RAS blocker includes angiotensin converting enzyme (ACE) inhibitors and Angiotensin receptor blockers (ARBs)

GFR glomerular filtration rate 
Table 2 The association between KIM-1/creatinine and chronic heart failure: Multivariable Cox regression models

\begin{tabular}{|c|c|c|c|c|c|}
\hline & \multirow{2}{*}{$\begin{array}{c}\text { Number of } \\
\text { events / } \\
\text { numbers } \\
\text { at risk }\end{array}$} & \multirow[t]{2}{*}{ Incidence rate } & \multirow{2}{*}{$\begin{array}{c}\text { Model A } \\
\text { Hazard ratio } \\
(95 \% \mathrm{CI}) \\
\end{array}$} & \multirow{2}{*}{$\begin{array}{c}\text { Model B } \\
\text { Hazard ratio } \\
(95 \% \mathrm{CI}) \\
\end{array}$} & \multirow{2}{*}{$\begin{array}{c}\text { Model C } \\
\text { Hazard ratio } \\
(95 \% \mathrm{CI})\end{array}$} \\
\hline & & & & & \\
\hline First tertile $(<67 \mathrm{ng} / \mathrm{mmol})$ & $19 / 190$ & $\begin{array}{c}1.23 \\
(0.84-2.08)\end{array}$ & referent & Referent & referent \\
\hline Second tertile $(67-128 \mathrm{ng} / \mathrm{mmol})$ & $23 / 188$ & $\begin{array}{c}1.30 \\
(1.13-2.55)\end{array}$ & $\begin{array}{c}1.30 \\
(0.71-2.39)\end{array}$ & $\begin{array}{c}1.42 \\
(0.77-2.62)\end{array}$ & $\begin{array}{c}1.38 \\
(0.75-2.54)\end{array}$ \\
\hline Third tertile $(>128 \mathrm{ng} / \mathrm{mmol})$ & $31 / 187$ & $\begin{array}{c}2.35 \\
(1.75-3.54)\end{array}$ & $\begin{array}{c}1.99 * \\
(1.12-5.53)\end{array}$ & $\begin{array}{c}1.89 * \\
(1.06-3.39)\end{array}$ & $\begin{array}{c}1.81^{*} \\
(1.01-3.29)\end{array}$ \\
\hline
\end{tabular}

Model A age

Model B age, established cardiovascular risk factors

Model $\mathrm{C}$ age, established cardiovascular risk factors, GFR, urinary albumin/creatinine 
Table 3 Data portraying the interplay between KIM-1/creatinine and GFR and the risk for chronic heart failure: Cox regression

\begin{tabular}{lccc}
\hline & $\begin{array}{c}\text { Number of } \\
\text { events } / \\
\text { numbers at } \\
\text { risk }\end{array}$ & Model A & Model B \\
& & HR & HR \\
& & (95\% CI) & referent \\
Normal GFR and normal KIM-1/creatinine & $32 / 320$ & referent & 1.34 \\
Normal GFR and high KIM-1/creatinine & $18 / 135$ & 1.47 & $(0.74-2.43)$ \\
Low GFR and normal KIM-1/creatinine & $10 / 56$ & $2.10 *$ & 1.76 \\
Low GFR and high KIM-1/creatinine & $13 / 54$ & $(1.03-4.27)$ & $(0.84-3.67)$ \\
& & $(1.93-7.04)$ & $3.26 \ddagger$ \\
\hline
\end{tabular}

Normal GFR $\left(\geq 60 \mathrm{~mL} / \mathrm{min} / 1.72 \mathrm{~m}^{2}\right)$ and normal KIM-1/creatinine $(\leq 128 \mathrm{ng} / \mathrm{mmol})$

Model A age

Model B age, established cardiovascular risk factors

$* \mathrm{p}<0 . \mathrm{p} 05,+\mathrm{p}<0.001$ 
Figure 1. Association Between Urinary KIM-1/creatinine and Heart Failure Incidence Assessed Via Regression Splines. T= Tertiles

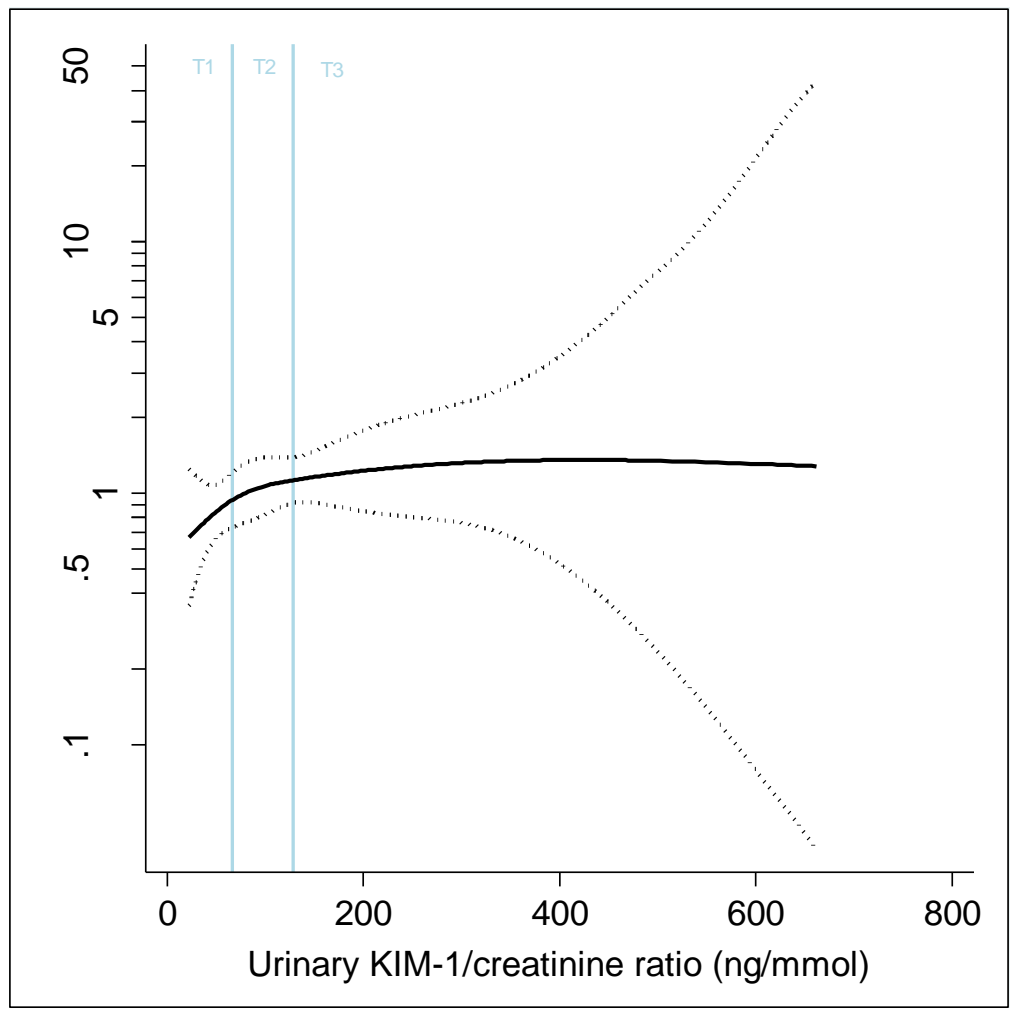


Figure 2. Cumulative Incidence of Heart Failure Morbidity by tertiles of the KIM$1 /$ creatinine

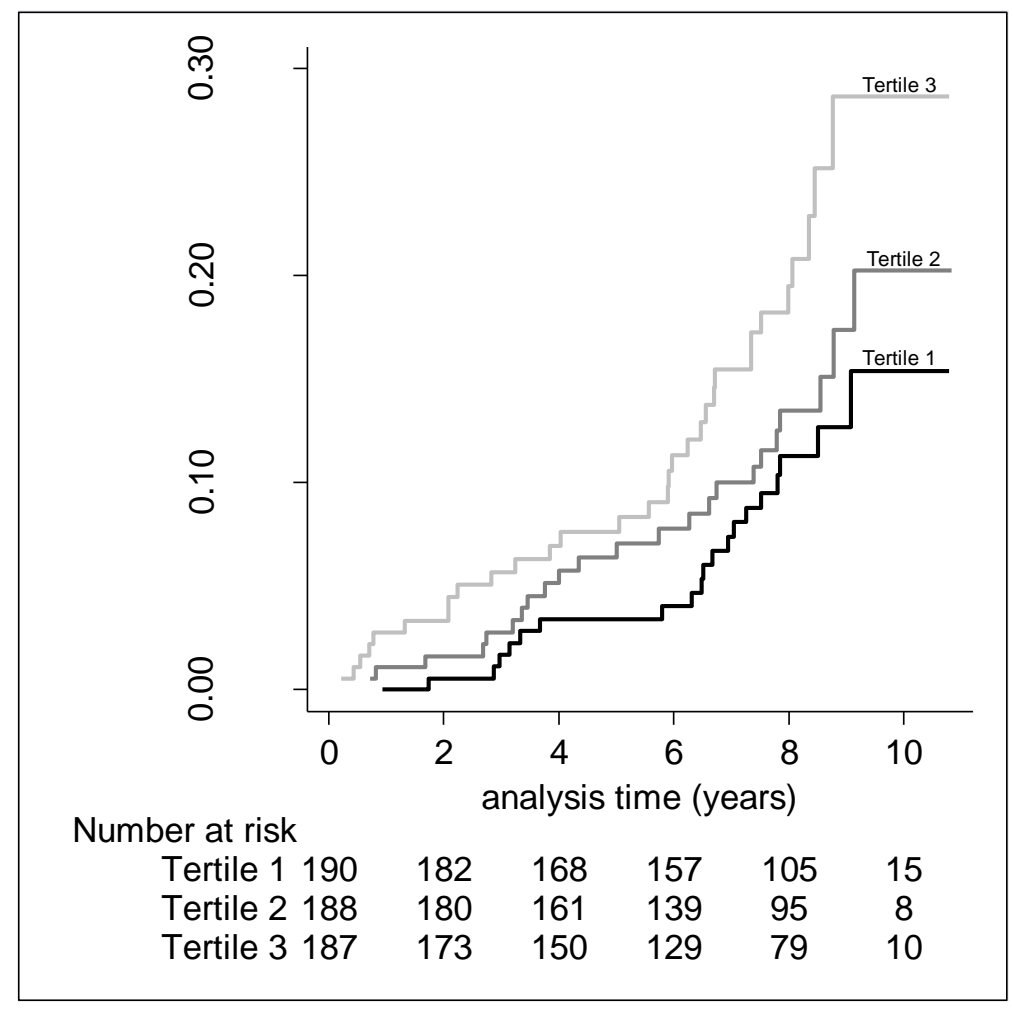

